\title{
Migração Reversa no Tempo (RTM) de Dados Sísmicos Adquiridos com Fonte do Tipo Boomer
}

\author{
Laian De Moura Silva*(CPGG/IGEO/UFBA) e Marco Antônio B. Botelho (CPGG/IGEO/UFBA)
}

Copyright de 2016, SBGf - Sociedade Brasileira de Geofísica.

Este texto foi preparado para a apresentação no VII Simpósio Brasileiro de Geofísica Ouro Preto, 25 a 27 de Outubro de 2016. Seu conteúdo foi revisado pelo Comite Técnico do VII SimBGf, mas não necessariamente representa a opinião da SBGf ou de seus associados. É proibida a reprodução total ou parcial deste material para propósitos comerciais sem prévia autorização da SBG.

\section{Abstract}

The present study investigates the application of Reverse Time Migration (RTM) on high frequency seismic line acquired using boomer source. These seismic sections are mono channel and it is impossible to use the CMP thecnique to define one velocity field. It is presented one interactive procedure using RTM based on reflector shape and hyperbole collapses, which starts with one migration using the water sonic velocity $(1500 \mathrm{~m} / \mathrm{s})$. It is followed by a second migration model defined by the water layer limited below by the migrated sea floor shape. This procedure is repeated with different velocities until to complet the velocity macro model, allways controlling the correct velocity with the collapses of the hyperboles below. The study area is situated on flamengo bay on the Ubatuba coast São Paulo where was acquired six (6) lines using the boomer source (10000 Hz).

\section{Resumo}

O presente estudo investiga a aplicação da migração reversa no tempo na linha sísmica de alta frequência adquiridos utilizando fonte boomer. Essas seções sísmicas são mono canal é impossível o uso da técnica CMP para definir um campo de velocidade. Apresentase um procedimento interativo usando RTM baseada no colapso de hipérbole, que começam com uma migração usando o velocidade do som na água $(1500 \mathrm{~m} / \mathrm{s})$. Ele é seguido por um segundo modelo de migração definido pela camada de água limitada pela forma abaixo do fundo do mar migrado. Este procedimento é repetido com diferentes velocidades de até completar o modelo de macro velocidade, sempre controlando a velocidade correta que colapsa as hipérboles. A área de estudo situase na região de Baia de Flamengo na costa Ubatuba São Paulo onde foram adiquiridas (6) linhas usando a fonte boomer $(10000 \mathrm{~Hz})$.

\section{Introdução}

Levantamento de dados de fonte tipo boomer, é uma modalidade de sísmica mono-canal (afastamento fontereceptor nullo). Ou seja, esses dados se assemelham a um dado sísmico convencional já empilhado, logo todo o processamento é feito no dominio do traços, semelhante ao cpd nos métodos convencionais. Este trabalho mostra que é possível migrar dados de sísmica rasa a velocidade constante e que seus resultados são satisfatórios. Para migrar os dados foi usado a Migração Reversa no Tempo com operadores de diferenças finitas de segunda ordem no tempo e quarta ordem no espaço.

\section{Princípio de Funcionamento da Fonte do Tipo Boomer}

As fontes acústicas impulsivas são aquelas que liberam grande quantidade de energia num intervalo de tempo que idealmente é próximo de zero. O boomer é uma fonte sísmica impulsionada eletromagneticamente que consiste em um banco de capacitores que geram uma descarga eletrica através de uma bobina plana. Correntes de Foucault são introduzidas em uma placa de cobre ou alumínio que são mantido sobre uma bobina que fica apoiada sobre uma mola ou um amortecedor de borracha.

A interação entre o campo primário e secundário resultantes causam uma repulsão da placa rapidamente pela bobina. A queda de pressão repentina produz na água um campo de pressão com amplo espectro de frequência. Este fontes podem absorver até $1000 \mathrm{~J}$ de energia e, portanto, tornarem viável a emissão de sinais de um amplo espectro de frequências (p.ex. 500 a 20.000 $\mathrm{Hz}$ ) com alta potência.

Boomers estão entre as principais fontes acústicas utilizadas na investigação de áreas rasas, pois este tipo de fonte emite amplo espectro de frequências, possibilitando resolução centimétrica em algumas situações, com alta energia, largos pulsos em relação a outras fontes de sísmica rasa. Na Figura(1) podemos observar a relação entre as fontes e as frequência geradas por cada tipo.

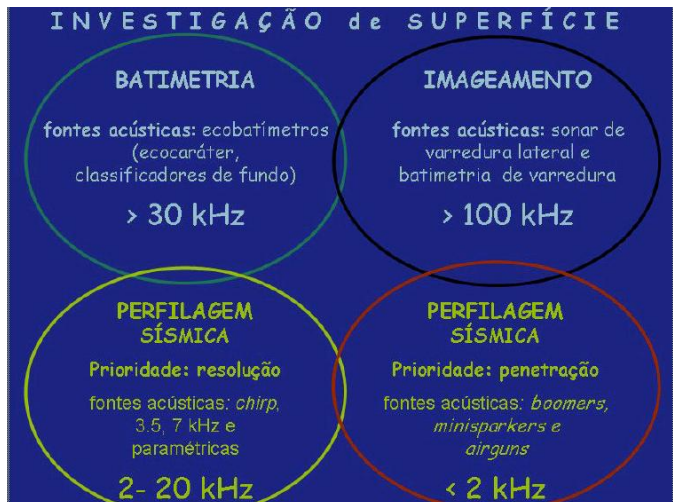

Figure 1: Fontes acústicas e suas frequências geradas por (de Souza,2006)

\section{Migração RTM}

O Método RTM(Reverse Time Migration), é método de migração mais completo e robusto que os espectrais 
por assumir qualquer variação lateral de velocidade, e com isso, sendo capaz de imagear regiões de geologia complexa, este processo ocorre pela depropagação no tempo, do campo de ondas, calcula-se a posição espacial dos pontos de reflexão do campo de ondas em profundidade, usando a condição de imagem, correspondente a $\mathrm{t}=0$. Ao final do processo de migração a seção em tempo $S(x, z=0, t)$ fornecerá seção e profundidade $S(x, z, t=0)$ corresponente ao modelo geométrico /geológico. Os dado sísmicos de fonte do tipo boomer se assemelham a dados empilhados (offset nullo) e com isso o método usado será o RTM pósempilhamento.

Para isso, supõem-se que toda energia presente em um determindado tempo $t=t_{d}$ no dado sísmico pósempilhado é proveniente de uma reflexão no tempo $t=$ $t_{d} / 2$. Desta forma, o coeficiente de reflexão deste refletor pode ser determinado depropagando-se esta energia até o tempo $t=t_{d} / 2$ (considerando as velocidades reais do modelo), ou, equivalente a depropagar a energia até o tempo $\mathrm{t}=0$ (considerando as velocidade de propagação como sendo a metade das velocidade reais do modelo).

$\mathrm{Na}$ migração reversa no tempo pós-empilhamento, supões-se que os dados sísmicos reais se comportam como se fosse oriundos de uma modelagem sísmica, considerando o modelo do refletor explosivo (Loewenthalet al.,1983), sendo os dados depropagados até o tempo $t=0$. A imagem em profundidade é obita diretamente do campo de onda no instante $t=0$, ou seja, ao final do processo de depropagação tem-se a imagem em profundidade dos refletores em subsuperfície.

O RTM utiliza a seção sísmica real como condição de contorno e realiza a migração no plano objeto, calculando, a partir do tempo final da seção, o campo de onda para cada tempo até o tempo igual a zero. A seção em profundidade em $\mathrm{t}=0$ representa a seção migrada, $P(x, z, t=0)$. utilizando a equação completa da onda para as derivadas espaciais e de segunda ordem para as derivadas em relação ao tempo. A equação realiza a marcha reversa no tempo é dada por:

$$
\begin{aligned}
P_{m, j}^{n-1}= & \left\{2-2,5\left(A_{x}+A_{z}\right)\right\} P_{m, j}^{n}-P_{m, j}^{n+1}+ \\
& \frac{A_{x}}{12}\left\{16\left(P_{m-1, j}^{n}+P_{m+1, j}^{n}\right)-\left(P_{m-2, j}^{n}+P_{m+2, j}^{n}\right)\right\}+ \\
& \left\{\frac{A_{z}}{12}\left\{16\left(P_{m, j-1}^{n}+P_{m, j+1}^{n}\right)-\left(P_{m, j-2}^{n}+P_{m, j+2}^{n}\right)\right\}\right.
\end{aligned}
$$

O processo inicia-se como o campo de onda totalmente zerado para $t>t_{f}$, onde $t_{f}$ representa o tempo final da seção sísmica. Para $t=t_{f}$, apenas a primeira linha será diferente de zero, ou seja $P\left(x, z=0, t=t_{f}\right) \neq=0$, e corresponde aos valores com tempo máximo na seção registrada. Aplicando a marcha reversa no tempo, pode-se calcular os valores do campo das ondas ascendentes para cada tempo, o qual corresponde ao instante que ocorreu um conjunto de reflexões; tornando possível calcular $P_{i}\left(x, z, t=t_{i}\right)$, com i variando de $t_{f} / s_{r}$, onde $s_{r}$ é igual ao intervalo de amostragem, até o instante zero, onde $t_{0}=$ 0 , tendo como condição de contorno a seção registrada $P(x, z=0, t)$.

\section{Área de Estudo}

A área de estudo, onde estão localizadas as 2 linhas sísmica de fonte do tipo boomer (Figura2), é conhecida como Boqueirão da llha de Anchieta uma depressão localizada entre as baías de flamengo e palmas na região de Ubatuba São Paulo. O boqueirão possui profundidade máxima de nível do mar em torno de $35 \mathrm{~m}$ e 500 metros de largura.

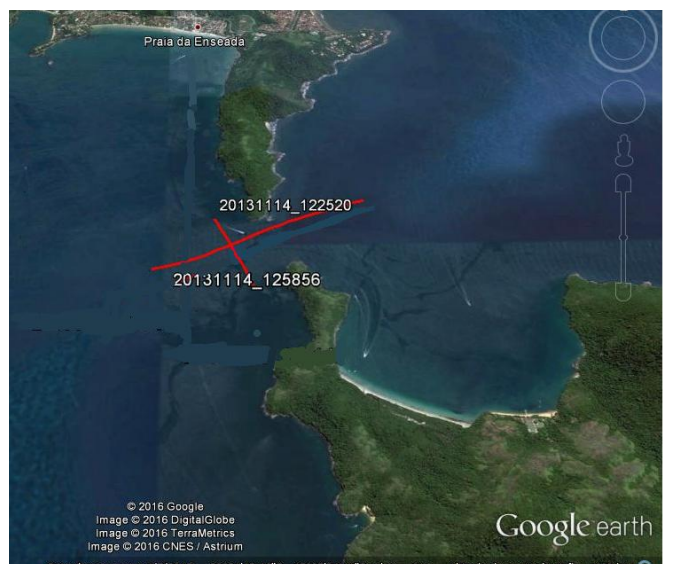

Figure 2: Imagem da região deo Boqueirão,Ubatuba,SP. (Imagens geradas pelo Google Eart)

A origem do boqueirão esta provavelmente relacionado a um antigo sistema de drenagem desenvolvido durante o quaternário superior no periodo de baixo nivel do mar assosiado a estruturas tectonicas (Almeida, apud Mahiques e Souza,1999). Já (Mahiques et al.,apud Mahiques e Souza,1999) considera que a evolução do boqueirão esta associado a erosão por corrente de maré durante o aumento do nível do mar em Santos.

Ao estudar a dinâmica de fundo da enseada do Flamengo (Ubatuba-Sp) usando identificaccão de foraminíferos (Bonfante e Mahiques,2005) encontrou próximo a região de Boqueirão de Ilha de Anchieta, foraminíferos bioindicadores de ambiente rico em materia orgânica. $\quad \mathrm{Na}$ tabela(1) podemos ver as posições geográficas das linhas.

\begin{tabular}{|c|c|}
\hline Linha No & Posição das Linhas \\
\hline $\mathrm{L}-125856$ & $23^{\circ} 31^{\prime}$ a $23^{\circ} 31^{\prime} \mathrm{S} / 45^{\circ} 05^{\prime}$ a $45^{\circ} 05^{\prime} \mathrm{O}$ \\
\hline $\mathrm{L}-122520$ & $23^{\circ} 31^{\prime}$ a $23^{\circ} 31^{\prime} \mathrm{S} / 45^{\circ} 04^{\prime}$ a $45^{\circ} 05^{\prime} \mathrm{O}$ \\
\hline
\end{tabular}

Table 1: Localização das Linhas

\section{Processamento das Linhas}

A descrição das etapas do processamento, será melhor representada através de um fluxograma (Figura3) e os detalhes do fluxo serão discutidos abaixo.

Os parâmetros das linhas L-125856 e L-122520 estão descritos nas tabela2 e tabela3 respectivamente.

A linha L-125856 após leitura, aplicação do filtro trapezóidal do tipo passa-banda (Figura4) com as seguintes configurações $f 1=50$ f2=70 f3=1500 f4=1600 e após ganho para compensar a perca de amplitude, causado pelo fenômeno de divergência esférica e absorção do sinal sísmico, podemos ver na Figura(5). As regiões em vermelho 1 e 2 mostrada na figura são regiões da linha sísmica, será comparada após migração.

Já a Figura(6) representa a linha L-122520 após filtro 


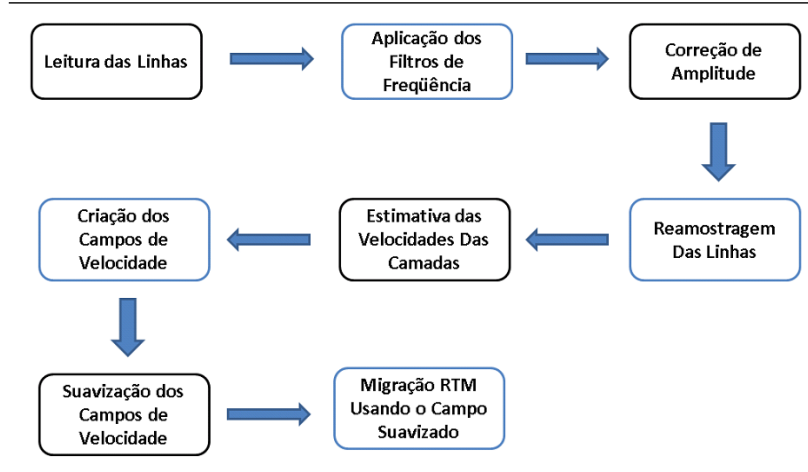

Figure 3: Fluxo Processamento

\begin{tabular}{|c|c|}
\hline No de traços (nx) & 939 \\
\hline No de amostra (nz) & 2801 \\
\hline No de amostra em tempo (ns) & 5602 \\
\hline Amostragem em profundidade dz (m) & 0.03927 \\
\hline Amostragem em tempo dt (s) & 0.000025 \\
\hline Amostragem entre os traços (m) & 0.954 \\
\hline
\end{tabular}

Table 2: Tabela com os parametros da migração da linha L-125856

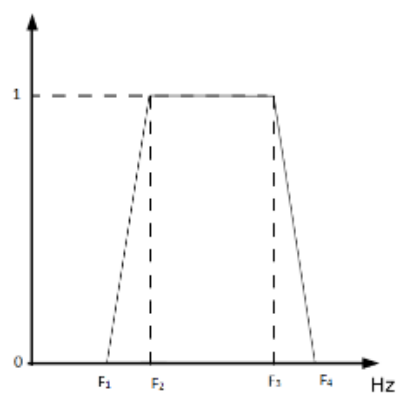

Figure 4: Filtro Trapezóidal do Tipo Passa Banda

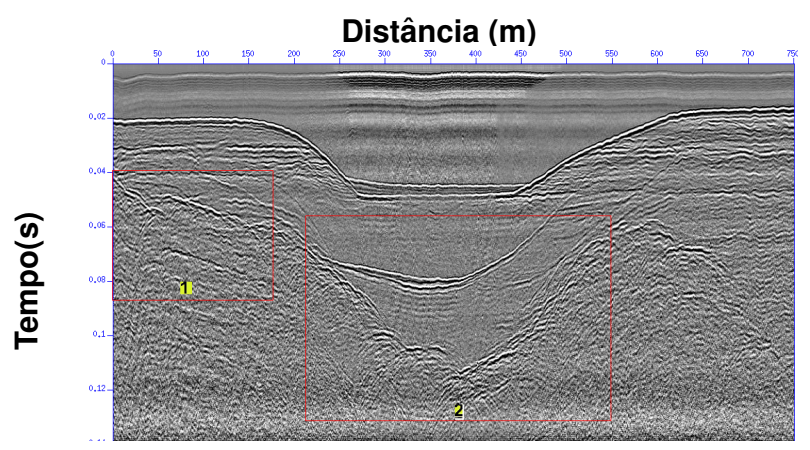

Figura 5: Linha L-125856 Após processamento básico

passa-banda com a configuração $f 1=20 \quad f 2=50 \quad f 3=1400$ $\mathrm{f} 4=1500$ (Figura4) e coreção de amplitude assim como na linha L-125856. Novamente foi separada na figura duas regiões (em vermelho) para fins de comparação com a linha migrada na seção de resultado.

\section{Resultados}

A RTM necessita atender a condição de estabilidade que é dada por: $A_{x}+A_{z} \leq \frac{3}{4}$ vide Faria(1980). Para isso é necessário reamostrar o dado sísmico. O dado foi

\begin{tabular}{|c|c|}
\hline No de traços (nx) & 2499 \\
\hline No de amostra (nz) & 2801 \\
\hline No de amostra em tempo (ns) & 5602 \\
\hline Amostragem em profundidade dz (m) & 0.042 \\
\hline Amostragem em tempo dt(s) & 0.000025 \\
\hline Amostragem entre os traços & 0.800 \\
\hline
\end{tabular}

Table 3: Tabela com os parametros da migração da linha L-122520

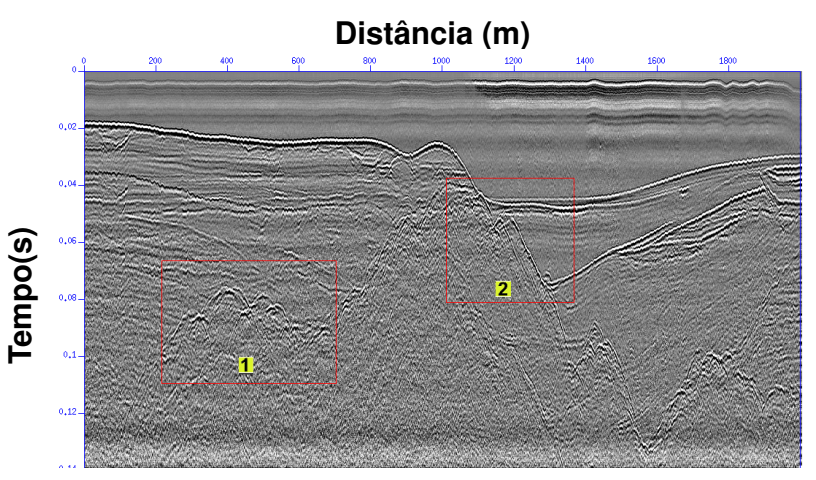

Figura 6: Linha L-122520 Após processamento básico

reamostrado para dobrar o número de amostras estes (ns=5602) e conservam o número de traços. Após estes procedimentos, os dados foram migrado inicialmente com a velocidade constante de $1500 \mathrm{~m} / \mathrm{s}$, seu resultado gerou a interfacie do assoalho marinho na profundidade correta.

Numa segunda migração o campo de velocidade foi aprimorado com atribuição de $1500 \mathrm{~m} / \mathrm{s}$ para primeira camada, e mudando o valor da velocidade da segunda camada até encontrar a velocidade que melhor imageava o refletor abaixo do assoalho marinho (segunda camada), tendo como controle o colapso de hipérboles, e assim para determinar a velocidade da terceira camada.

O campo de velocidade da linha L-125856 podemos ver na (Figura7), os valores das velocidades estão com seu valor pela metade devido o método do refletor explisivo (lowental citar).

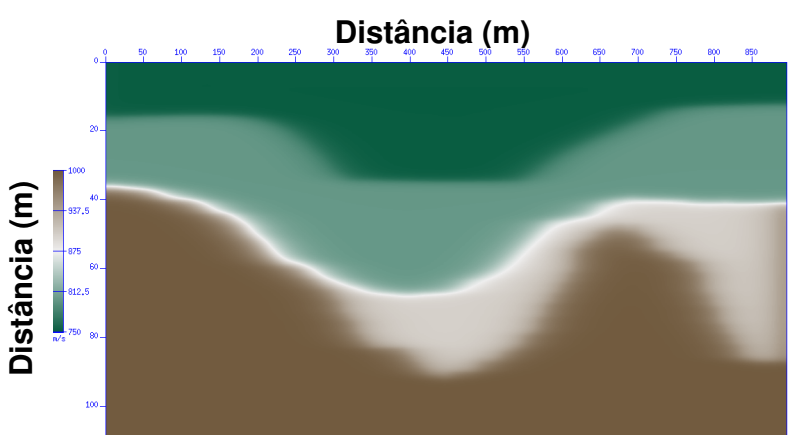

Figura 7: Campo de velocidade da Linha L-125856

Assim também está o campo da linha L-122520 representado na (Figura8). Os dois campos de velocidade estão suavizados para que não haja variações bruscas de velocidade, pois estas variações prejudicam a resolução 
da seção final.

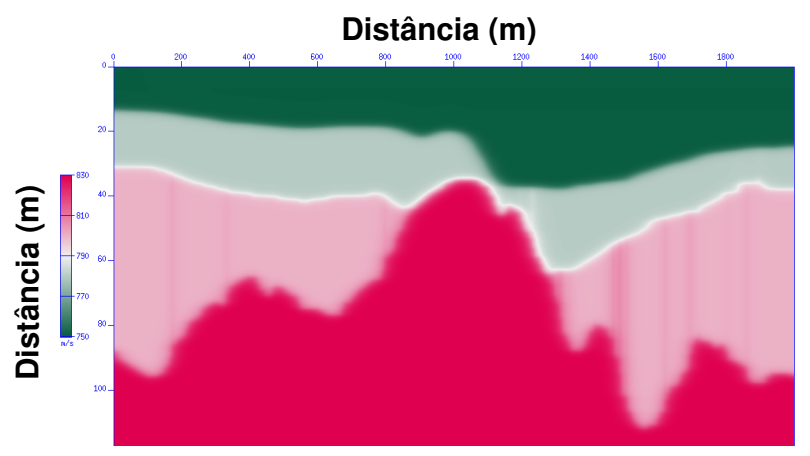

Figura 8: Campo de velocidade da Linha L-122520

O resultado da migração da linha L-125856 podemos ver na (Figura9) em destaque nesta figura temos duas regiões em vermelho que para observarmos melhor o resultado do colapso das hipérbolas nas camadas selecionadas. $\mathrm{Na}$ (Figura10) vemos um detelhe maior das duas regiões da linha L-125856 antes e depois da migração

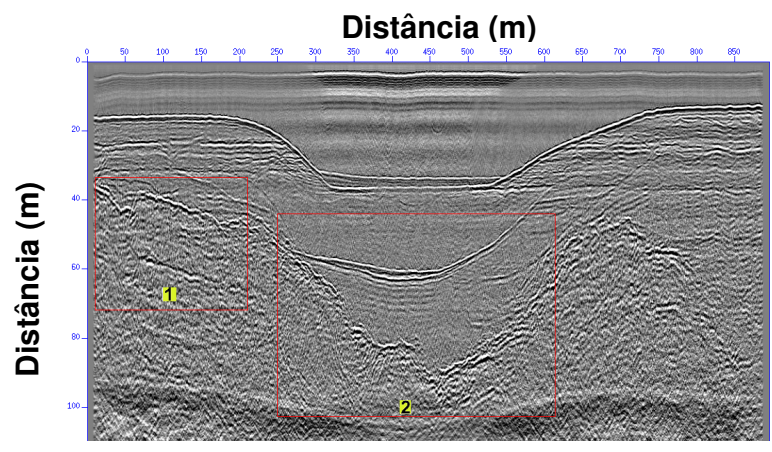

Figura 9: Resultado da migração da Linha L-125856

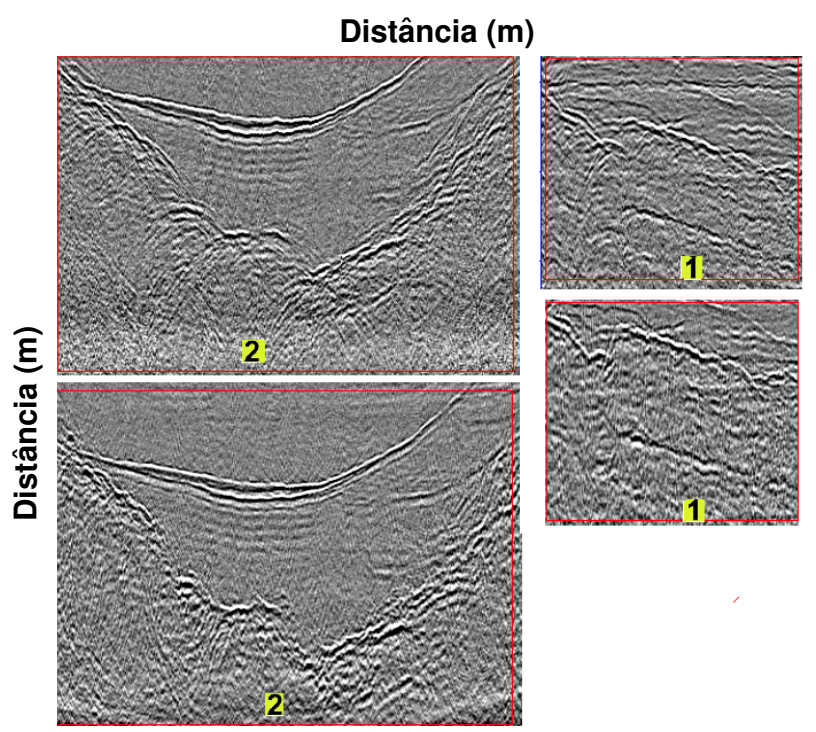

Figura 10: Comparação entra antes e depois da migração da Linha L-125856
Para a linha L-122520 o resultado da migração vemos na (Figura11) e na (Figura12) podemos ver com maior detalhe a região em vermelho, nesta região vemos como a migração colapsou bem as hipérboles.

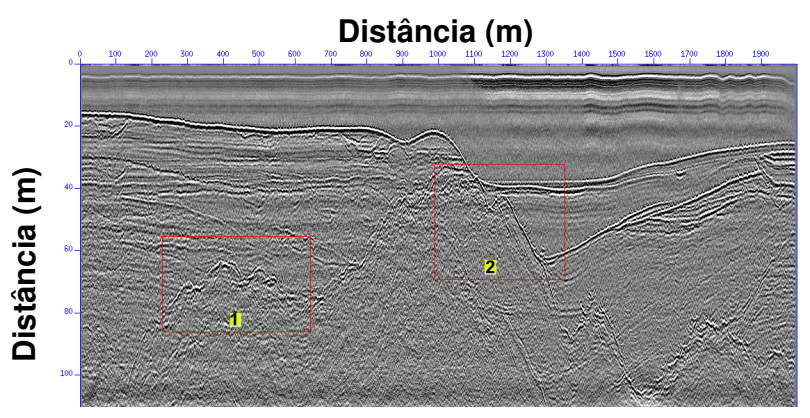

Figura 11: Resultado da migração da Linha L-122520

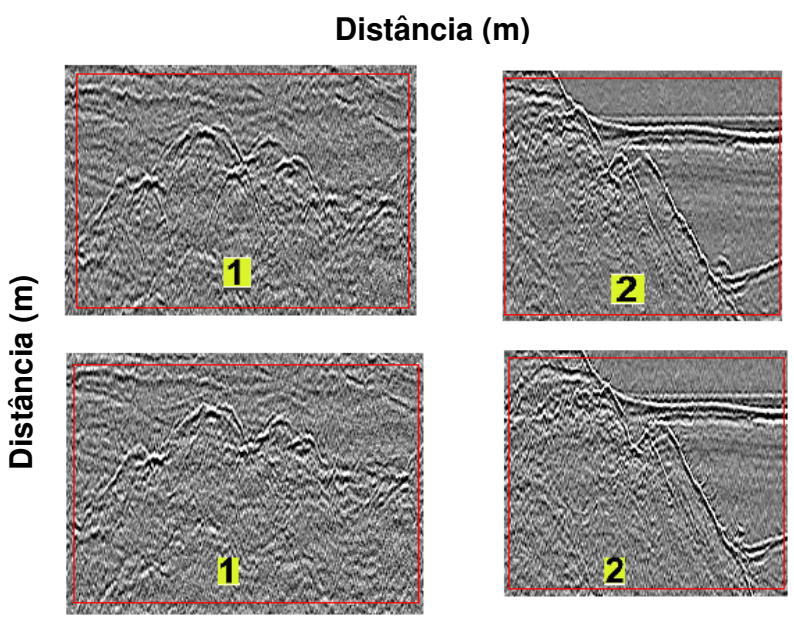

Figura 12: Comparação entra antes e depois da migração da Linha L-122520

\section{Conclusões}

Este trabalho mostra que é possível usar a migração RTM em dados de sísmica rasa de alta frequência mono canal. O campo de velocidade pode ser obtido por meio de várias migrações interativas, iniciando com a camada dágua. Existindo eventos de difrações bem definidos na seçõ sísmica em diferentes profundidades é possível estabelecer uma segunda, terceira ou mesmo mais camadas sempre se baseando no colapso das hipérboles. O processo inicia com a camada dágua $(\mathrm{v}=1500 \mathrm{~m} / \mathrm{s})$ e a geometria do assoalho marinho e vai estabelecendo as novas camadas em sentido crescente como profundidade.

Podemos concluir sobre a melhor continuidade do refletor correspondente ao topo do embasamento onde a RTM efetua o completo colapso das hipérboles, graças a determinação de um campo de velocidade próximo á realidade usando a ferramenta RTM. 


\section{Agradecimentos}

Os autores agradecem ao CPGG-UFBA pelo apoio no desenvolvimento deste trabalho. Agradecem ao instituto oceanográfico da usp e ao geofísico Luiz Antônio que permitiu a participação do segundo autor na campanha de treinamento de utilização do sistema Maridata nas costas de Ubatuba. Finalmente. Os autores agradecem ao termo de cooperação número 4600302960 assinado por Petrobras (Cenpes)-UFBA-FAPEX, o qual permitiu a compra da fonte sísmica Boomer e dos componentes de registro, o que permitiu esta pesquisa.

\section{Referências}

de Souza, Luiz Antonio Pereira. Revisão crítica da aplicabilidade dos métodos geofÃsicos na investigação de áreas submersas rasas. Diss. Universidade de São Paulo, 2006.

MAHIQUES, MM de. "Variaçẽs temporais na sedimentação holocênica dos embaiamentos da região de Ubatuba (SP)."Unpublished PhD Dissertation thesis, Instituto Oceanográfico da Universidade de São Paulo (1992).

Botelho, MARCO ANTONIO BARSOTTELLI. "Modelamento Sísmico na Bacia do Recôncavo usando a Técnica de Traçamento de Raios."Modelamento Sísmico na Bacia do Recôncavo usando a Técnica de Traçamento de Raios (1986).

Loewenthal, D., et al. "The wave equation applied to migration."Geophysical Prospecting 24.2 (1976): 380-399.

Faria, Eduardo Lopes. "Migração antes do empilhamento utilizando propagação reversa no tempo."Dissert. de Mestrado, UFBA (1986). Mahiques, Michel Michaelovitch de, and Luis Antonio Pereira de Souza. "Shallow seismic reflectors and upper Quaternary sea levei changes in the Ubatuba region, São Paulo State, Southeastern Brazil."Revista Brasileira de Oceanografia 47.1 (1999): 01 10.

LanÃ one, RAFAELA BONFANTE, W.Â. N. I. A. Duleba, and MICHEL M. Mahiques. "Dinâmica de fundo da enseada do Flamengo, Ubatuba, Brasil, inferida a partir da distribuição espacial, morfometria e tafonomia de foraminíferos."Revista Brasileira de Paleontologia 8.3 (2005): 181-192. 